\title{
NON-ANALYTICITIES IN THREE-DIMENSIONAL GAUGE THEORIES
}

\author{
M. ASOREY*, D. GARCIA-ALVAREZ \\ Departamento de Física Teórica, Universidad de Zaragoza, 50009 Zaragoza, Spain. \\ J.L. LÓPEZ \\ Departamento de Matemáticas e Informática, Universidad Pública de Navarra, 31006 \\ Pamplona, Spain.
}

\begin{abstract}
Quantum fluctuations generate in three-dimensional gauge theories not only radiative corrections to the Chern-Simons coupling but also non-analytic terms in the effective action. We review the role of those terms in gauge theories with massless fermions and Chern-Simons theories. The explicit form of non-analytic terms turns out to be dependent on the regularization scheme and in consequence the very existence of phenomena like parity and framing anomalies becomes regularization dependent. In particular we find regularization regimes where both anomalies are absent. Due to the presence of non-analytic terms the effective action becomes not only discontinuous but also singular for some background gauge fields which include sphalerons. The appearence of this type of singularities is linked to the existence of nodal configurations in physical states and tunneling suppression at some classical field configurations. In the topological field theory the number of physical states may also become regularization dependent. Another consequence of the peculiar behaviour of three-dimensional theories under parity odd regularizations is the existence of a simple mechanism of generation of a mass gap in pure Yang-Mills theory by a suitable choice of regularization scheme. The generic value of this mass does agree with the values obtained in Hamiltonian and numerical analysis. Finally, the existence of different regularization regimes unveils the difficulties of establishing a Zamolodchikov c-theorem for three-dimensional field theories in terms of the induced gravitational Chern-Simons couplings.
\end{abstract}

\footnotetext{
${ }^{*}$ I am one of the very fortunate persons who had deep scientific and vital resonances with Ian Kogan. Still under the effect of the tragedy, in June 2003, I promised to Ian and to myself to finish a joint paper which we had outlined few weeks before. There is an old spanish popular aphorism telling that lo que no puede ser, no puede ser, y además es imposible (what cannot be, cannot be, and furthermore it is impossible!). Now I better understand the meaning of the tautological aphorism, without Ian's resonances I will not be able to accomplish my promise. I am sorry, Ian.
} 


\section{Introduction}

In $2+1$ dimensions the number of degrees of freedom of massive and massless relativistic particles is the same. This peculiar behaviour permits a smooth transition from massless to massive regimes in the same theory without the need of extra fields. In gauge theories this transition can be simply achieved by the addition of a Chern-Simons term to the ordinary Yang-Mills action [1]. For the same reasons there is no protection against the existence of radiative quantum corrections which either generate or suppress the topological mass.

The special characteristics of Chern-Simons term and its peculiar transformation law under large gauge transformations requires the quantization of its coupling constant $k \in \mathbb{Z}$ when the gauge group is compact. The constraint arises in the covariant formalism as a consistency condition for the definition of the euclidean functional integral due to the special transformation properties of the Chern-Simons action under large gauge transformations [1]. In the canonical formalism it appears as a necessary condition for the integration of Gauss law on the physical states [2][3]. Both interpretations are based on non-infinitesimal symmetries and therefore the quantization condition can not be inferred from perturbative arguments. However, unexpectedly the perturbative contributions of quantum fluctuations do not seem to change the integer nature of the Chern-Simons coupling constant in most of standard renormalization schemes [4]-[10]. From a pure quantum field theory point of view this behavior is bizarre because in absence of perturbative symmetry constraints there must always exist regularization schemes where the effective values of the coupling constants of marginal local terms are arbitrary. Indeed, such regularization schemes exist but require a fine tuning of the leading ultraviolet behavior of parity even and parity odd terms of regulators [10].

The perturbative quantum corrections are not the only contributions of quantum fluctuations. There exist additional contributions to the effective gauge action which cannot be obtained in perturbation theory because they are not analytical on gauge fields. The presence of such non-analytic contributions in one loop approximation is more evident in the case of regularizations which do not preserve the integer value character of the effective Chern-Simons coupling. They appear as necessary to compensate the 
anomalous transformation law of Chern-Simons terms under large gauge transformations. The role of those terms is crucial to understand the finite temperature behaviour of gauge theories in $2+1$ dimensions [13]-[16]. They are similar to the well known non-analytic terms which appear in the $\eta$ invariant of the spectral asymmetry [11] of the operator $* d_{A}+d_{A} *$ induced by the changes of signs in the spectral flow [12].

The study of non-analytic terms of the effective action and their physical implications is the main goal of our analysis. The discontinuities associated to these terms yield singularities which in the case of Chern-Simons theory seem to be mere artifacts of perturbation theory. The origin of the singularities is the same that appears in ordinary gauge theories in presence of massless quarks in the fundamental representations. In this case the singularities do have a simple physical origin, the existence of zero-modes of Dirac operator.

The main result of the paper is the proof that this kind of non-analyticities are regularization dependent which provides a further support to the claim that different renormalization methods define in fact different physical theories. The perturbative corrections to the Chern-Simons coupling constant can also be different and depend on the regularization method but those differences can be compensated in general by the choice of different renormalization schemes. However, the presence of different non-analytic contributions cannot be changed by the choice of appropriate renormalization schemes. In some way this provides a physical meaning to the non-perturbative constraint that requires the coupling of Chern-Simons counterterms must be an integer value. The meaning of the restriction is that the analytic behaviour of the effective partition function cannot be changed by the renormalization scheme and provides a novel physical role to the choice of regularization method.

Parity anomaly and framing anomaly have a common origin in the existence of odd quantum effects. Because of their dependence on the regularization method it is possible, thus, to find out some regularization regimes where both anomalies are absent.

Finally, the regularization dependence of these phenomena is also responsible of the failure of simple attempts to define a Zamolodchikov's cfunction in terms of gravitational Chern-Simons terms in order to generalize of Zamolodchikov c-theorem for three-dimensional theories. 


\section{Chern-Simons theory}

In the limit of infinite topological mass the gauge theory reduces to a ChernSimons topological theory [17] governed by the action

$$
k S_{\mathrm{CS}}(A)=\frac{k}{4 \pi} \int_{M} \operatorname{Tr}\left(A \wedge d A+\frac{2}{3} A \wedge A \wedge A\right) \quad k \in \mathbb{Z}
$$

where the coupling constant $k$ must be an integer for compact groups to have a consistent quantization ${ }^{\mathrm{a}}$. Let us consider $S U(N)$ gauge field theories for simplicity.

The theory is superenormalizable also in this topological limit. In the Hamiltonian formalism divergences appear in the normalization of physical states and the hermitian product of the Hilbert space [19]. The removal of these divergences generates a shift in the renormalized Chern-Simons coupling constant $k_{\mathrm{R}}=k+N$. In the covariant formalism the propagator is very singular because of the large gauge symmetry of the theory originated by its topological character. In perturbation theory one way of improving the UV behaviour of the propagator without breaking gauge invariance is by introducing higher derivative regulating terms into the classical action, e.g.

$$
\begin{gathered}
S_{\Lambda}(A)=S_{\mathrm{CS}}(A)+S_{\mathrm{R}}^{+}(A) \\
S_{\mathrm{R}}^{+}(A)=\frac{\lambda_{+}}{\Lambda} \int_{M} \operatorname{Tr} F_{\mu \nu}(A)\left(\mathbb{I}+\frac{\Delta_{A}}{\Lambda^{2}}\right)^{m} F_{\mu \nu}(A),
\end{gathered}
$$

where $\Delta_{A}=d_{A}^{*} d_{A}+d_{A} d_{A}^{*}$ is the covariant laplacian. For large enough values of the exponent $m$ there are not UV superficial divergences in diagrams with more than one loop. However, one loop divergences need of an extra PauliVillars regularization [20]. The resulting one loop effective action has no divergences even after the removal of the ultraviolet regulator $\Lambda \rightarrow \infty$. The renormalized perturbative effective action is of the form

$$
\begin{gathered}
\Gamma^{\text {pert }}\left(A^{R}\right)=\Gamma_{R}\left(A^{R}\right)+i \Gamma_{I}\left(A^{R}\right) \\
\Gamma_{I}\left(A^{R}\right)=k_{\mathrm{R}} S_{\mathrm{CS}}\left(A^{R}\right)
\end{gathered}
$$

with $k_{\mathrm{R}}=k+N$. The first non-trivial contribution to $\Gamma\left(A^{R}\right)$ arises from the four point function [21].

\footnotetext{
${ }^{a}$ A generalization for non-compact gauge groups is straightforward [18].
} 
The Hamiltonian approach yields similar results, but this coincidence is not based on general symmetry principles. Thus, it should be possible the existence of a regularization where the renormalization of $k$ is not a simple shift of $k$ by $\mathrm{N}$ units. Indeed, there exist other gauge invariant regularizations, e.g. [10].

$$
\begin{gathered}
S_{\Lambda}(A)=S_{\mathrm{CS}}(A)+S_{\mathrm{R}}^{-}(A) \\
S_{\mathrm{R}}^{-}(A)=\frac{\lambda_{-}}{\Lambda^{2}} \int_{M} \operatorname{Tr} \epsilon^{\alpha \sigma \mu} F_{\alpha \nu}(A)\left(\mathbb{I}+\frac{\Delta_{A}}{\Lambda^{2}}\right)^{n} D_{A}^{\sigma}\left(\mathbb{I}+\frac{\Delta_{A}}{\Lambda^{2}}\right)^{n} F_{\mu \nu}(A)
\end{gathered}
$$

which after removing one-loop divergences yield an effective action like (2.1), but without radiative contributions to the effective value of the coupling constant $k_{\mathrm{R}}=k$. Even more general regularizations can be conceived, e.g.

$$
S_{\Lambda}(A)=S_{\mathrm{CS}}(A)+S_{\mathrm{R}}^{+}(A)+S_{\mathrm{R}}^{-}(A)
$$

In that case the result depends on the relative weights $\lambda_{-}>0$ and $\lambda_{+}>0^{\mathrm{b}}$ of $S_{\mathrm{R}}^{+}$and $S_{\mathrm{R}}^{-}$

$$
k_{\mathrm{R}}= \begin{cases}k+N & \text { if } m>2 n+1 / 2 \\ k+\frac{2 N}{\pi} \arctan \frac{\lambda_{+}}{\lambda_{-}} & \text {if } m=2 n+1 / 2 \\ k & \text { if } m<2 n+1 / 2\end{cases}
$$

In these very general regularization schemes the radiative corrections to the coupling constant present three different regimes which depend on the interplay between the ultraviolet behaviors of parity even terms $S_{\mathrm{R}}^{+}$of the regularized action and the parity odd terms of $S_{\mathrm{R}}^{-}$.

In the first regime the leading ultraviolet terms are parity even. The effective Chern-Simons coupling constant gets shifted by $N(k \rightarrow k+N)$, due to one loop gluonic radiative corrections. The third regime is characterized by an ultraviolet behavior dominated by parity odd terms and the absence of radiative corrections to $k$. In the transition regime parity even and parity odd terms have the same ultraviolet behavior and the quantum corrections to $k$ can take any real value which depends on the relative coefficients of the leading terms of parity even and parity odd interactions.

${ }^{\mathrm{b}}$ If $\lambda_{-}<0$ the results are slightly different [10] 
The phenomenon can be pictorially understood by looking at the way the shift of $k_{R}$ is generated. In fact,

$$
k_{R}=k+\frac{2 N}{\pi} \int_{0}^{\infty} \frac{d \Phi}{1+\Phi^{2}}=k+\frac{N}{\pi} \arctan \Phi(\infty)
$$

and the behaviour of

$$
\Phi=\frac{\lambda_{+} p\left(1+p^{2}\right)^{m}}{1+\lambda_{-} p^{2}\left(1+p^{2}\right)^{2 n}}
$$

is dictated by the form of $S_{\Lambda}(A)$

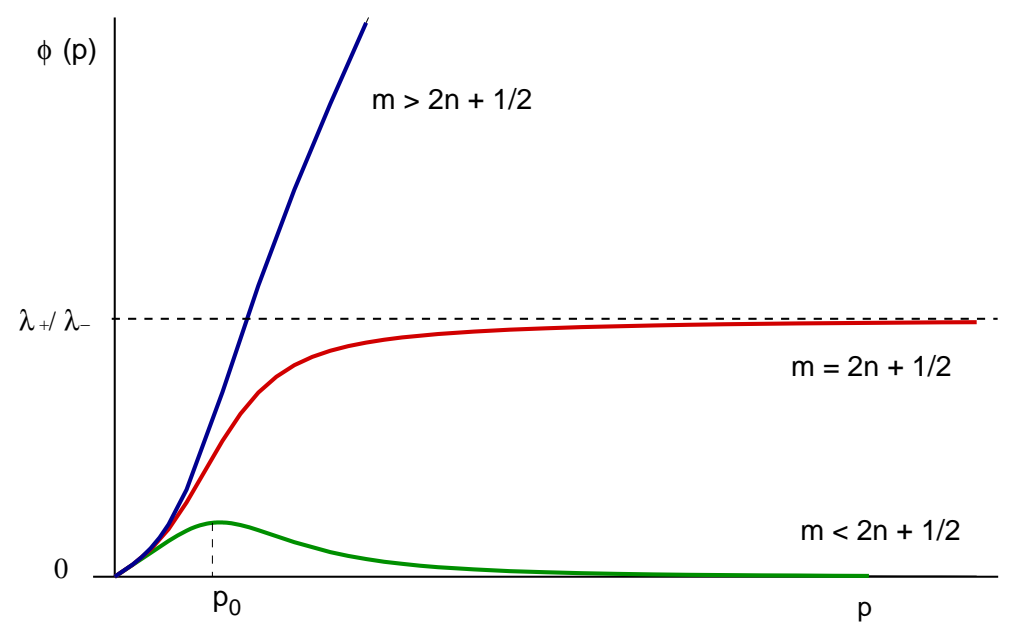

Figure 1. Behaviour of the function $\Phi$ for different regularization regimes

The actual value of the effective coupling constant can always be modified by a different choice of renormalization scheme because the Chern-Simons term is local and can be added as a conterterm. However, as pointed out in the previous section, the behaviour of Chern-Simons term under large gauge transformations requires that the bare coupling constant $k$ must be an integer number otherwise the quantum theory will be inconsistent, e.g. the functional integral will be ill defined. Such a constraint is a pure non-perturbative requirement, because large gauge transformations map small fields into large gauge fields and, therefore, they are genuine non-perturbative symmetries. In consequence, although in perturbation theory any local BRST invariant counterterm is valid, only counterterms which preserve the non-perturbative 
consistency condition can be added to the bare action. The condition imposes a very stringent constraint on counterterms which have to preserve the integer valued character of the bare coupling constant $k$. In particular, if the effective value of $k_{R}$ is not an integer, one cannot reduce the physical behavior of the system to the standard integer valued case by a consistent renormalization. Thus, the first and third regularization schemes are generic and equivalent from the physical point of view but the transition regime $m=2 n+\frac{1}{2}$ can not be reduced any of the other two regimes by the choice of a different scheme renormalization. In fact, the regime defines a new different theory.

In the generic case there is a correspondence of Chern-Simons states and the primary fields of rational conformal field theories [17]. In the transition regime the corresponding two-dimensional theory will be non-rational. In this sense, the transition regularization really defines a new type of theory,

\section{Parity Anomaly}

The existence of different regimes in the regularization of Chern-Simons theory opens new possibilities for the analysis parity anomaly.

This insight is further supported by the existence of a straightforward connection between one loop corrections of Chern-Simons theory and the determinant of a massless fermion in the adjoint representation [22]. Indeed, the second variation of Chern-Simons term and the corresponding ghost terms in a covariant Landau gauge yields an operator $S S_{A}$ which is equivalent to the square of Dirac operator $\left(D_{A}^{\text {ad }}\right)^{2}$ for adjoint fermions.

$$
\Delta_{A}=\left(\begin{array}{cc}
* d_{A} & d_{A} \\
d_{A}^{*} & 0
\end{array}\right) \approx\left(\not D_{A}^{\mathrm{ad}}\right)^{2}
$$

Therefore,

$$
\operatorname{det} \not D_{A}^{\mathrm{ad}}=\mathrm{e}^{-\frac{1}{2} \Gamma^{[1]}(A)}
$$

The effect of the existence of different regularization regimes is more intriguing because gauge invariance seems to be broken in the transition regime. 
Indeed, three different regimes can be generated by the following regularization of Dirac operator

$$
\not D_{A}^{\Lambda}=\not D_{A}+\lambda_{+} \frac{\not D_{A}^{2}}{\Lambda}\left(\mathbb{I}+\frac{\not D_{A}^{2}}{\Lambda^{2}}\right)^{m}+\lambda_{-} \frac{\not D_{A}^{3}}{\Lambda^{2}}\left(\mathbb{I}+\frac{\not D_{A}^{2}}{\Lambda^{2}}\right)^{2 n}
$$

with $\lambda_{ \pm}>0$ and the corresponding Pauli-Villars regulators. In that case the effective Chern-Simons coupling behaves in a similar way to the case of pure Chern-Simons theory.

$$
k_{\mathrm{R}}= \begin{cases}N & \text { if } m>2 n+\frac{1}{2} \\ \frac{2 N}{\pi} \arctan \frac{\lambda_{+}}{\lambda_{-}} & \text {if } m=2 n+\frac{1}{2} \\ 0 & \text { if } m<2 n+\frac{1}{2}\end{cases}
$$

If the fermions are in the fundamental representation of $S U(N)$ the result is analogous

$$
k_{\mathrm{R}}= \begin{cases}\frac{1}{2} & \text { if } m>2 n+\frac{1}{2} \\ \frac{1}{\pi} \arctan \frac{\lambda_{+}}{\lambda_{-}} & \text {if } m=2 n+\frac{1}{2} \\ 0 & \text { if } m<2 n+\frac{1}{2}\end{cases}
$$

Although the Pauli-Villars regularization method used here is completely gauge invariant also under large gauge transformation, gauge invariance under those transformations seems to be broken in the first two cases because the effective Chern-Simons term is not invariant. The puzzle is solved by noticing that the analytic perturbative radiative corrections do not exhaust all quantum corrections to the effective action. In fact, gauge invariance requires that the full radiative corrections must have a non-analytic conterpart which permit to recover full gauge invariance. Indeed,

$$
\Gamma\left(A^{R}\right)=\Gamma_{R}\left(A^{R}\right)+i \Gamma_{I}\left(A^{R}\right) \quad \text { with } \quad \Gamma_{I}(A)=k_{\mathrm{R}} S_{\mathrm{CS}}(A)+h(A)
$$

where $h(A)$ has a non-analytic dependence on $A$. However, in that case parity symmetry is not preserved at the quantum level because $S_{\mathrm{CS}}(A)$ is not invariant under parity symmetry whereas as it will be shown later $h(A)$ is parity invariant. This fact is on the origin of parity anomaly of threedimensional massless fermions. 
However, what is really intriguing is that in the third regime $m<2 n+1 / 2$ there is no parity anomaly because $k_{R}=0$ and the theory is at the same time invariant under global gauge transformations. This means that in fact, contrary to the common wisdom, the parity anomaly is not an unavoidable physical phenomena in a gauge invariant framework. A similar result is obtained with standard regularizations and infinite number of Pauli-Villars fields or lattice regularizations [23][24]. The results are reminiscent of those obtained by Slavnov [25] for the cancellation of the $S U(2)$ global Witten's anomaly in four-dimensional theories with chiral fermions in the fundamental representation [26]. The main difference between both results is that the Slavnov method requires an infinite number of Pauli-Villars regulating fields to cancel the anomaly in a gauge invariant way, whereas in this case a very simple UV modification of fermionic interactions yields a similar effect with a finite number of Pauli-Villars fields.

In the transition regime parity is also broken but the coefficient of the terms responsible for this phenomenon are different from those of the case where $m>2 n+1 / 2$. The ambiguity in the appearance or not of parity anomaly suggests that the effect looks more like a spontaneous symmetry breaking than a genuine anomaly breaking. Perhaps the phenomenon is nothing but a simple example of a more general feature on the breaking mechanism of discrete symmetries in three dimensions.

\section{Mass gap in Yang-Mills theory}

Indeed, the same phenomenon arises in the analysis of pure Yang-Mills theory

$$
S(A)=\frac{1}{2 g^{2}} \int_{M} \operatorname{Tr}|F(A)|^{2} .
$$

Using a similar regularization method which includes parity odd regulating terms and Pauli-Villars fields a Chern-Simons term can be induced in pure Yang-Mills theories. The infrared behaviour is dominated by the Yang-Mills term (4.1) which is parity even. The leading UV terms might be either a 
parity even term of the type $S_{R}^{+}(A)$ or a parity odd term like $S_{R}^{-}(A)$. The general result is

$$
k_{\mathrm{R}}= \begin{cases}0 & \text { if } m>2 n+\frac{1}{2} \\ -\frac{2 N}{\pi} \arctan \frac{\lambda_{+}}{\lambda_{-}} & \text {if } m=2 n+\frac{1}{2} \\ -N & \text { if } m<2 n+\frac{1}{2}\end{cases}
$$

In the first case no Chern-Simons coupling is generated whereas in the third case there is a non-trivial Chern-Simons radiative contribution with a coefficient $k_{R}=-N$. Both results follow from the behaviour of the flow displayed in Fig. 1. In the third case the non-trivial Chern-Simons term generates a topological mass

$$
m=\frac{g^{2} N}{2 \pi}
$$

which is in agreement with the actual value of the mass gap in pure YangMills theories [27][28]. The generation of a Chern-Simons term in the pure Yang-Mills theory points out the instability of the renormalization group flow. Moreover, it points toward a possible mechanism of generation of a mass gap in pure Yang-Mills theory. In this regime the theory is massive but parity symmetry is broken unlike the standard regime of Yang-Mills theory.

In the transition regime the theory gets a mass which depends on the relative weights of the leading parity even and parity odd terms.

\section{Non-analytic contributions}

The existence of non-analytic contributions to the imaginary part of the effective action $\Gamma_{I}^{[1]}(A)=k_{\mathrm{R}} S_{\mathrm{CS}}(A)+h(A)$ of massless fermionic determinants is known since the discovery of the spectral asymmetry and index theorem [11][29]. In the present case they are pointed out by the existence of Chern-Simons terms with non-integer coefficients [30][31]. The Pauli-Villars regularization method preserves gauge invariance and the only way to ensure the gauge invariance of the final result is by admitting the existence of a non-analytic contribution in $h(A)$ which transforms as

$$
h\left[A^{g}\right]=h[A]+2 \pi k_{R} n
$$

under large gauge transformations. 
The fermionic determinant $\not D_{A}$ is expected to have an analytic dependence on $A$ but the effective action is the logarithm of this determinant. The existence of a zero in the determinant induces a singularity in the effective action. Thus, the effective action $\Gamma_{A}$ diverges for (nodal) gauge configurations with fermionic zero modes. Every zero of an analytic function has an integer degree, which is measured by the discontinuity of the imaginary part of the corresponding logarithm. Thus, if the fermionic determinant has an analytic dependence on the background gauge field, the only possible discontinuities at a nodal configurations must by integer multiples of $\pi$ depending on the order of the zeros. For the simple zeros the value of the discontinuity through any continuous path of gauge fields crossing the nodal field is $\pi$. For double zeros the discontinuity is $2 \pi$ and so forth. If the trajectories of fields correspond to paths of three dimensional gauge fields induced by four-dimensional gauge fields with non-trivial topological charge $q$ it can be shown from the index theorem that the total discontinuity along the trajectory will be equal to $2 \pi q[26]$.

The regularized value of the fermionic determinant in the transition regime has an imaginary component of the effective actions which undergoes non-integer discontinuities. This fact signals an extra degree of nonanalyticity (no holomorphy) of the determinant in the transition regime and, thus, indicates that there is a radical difference of the transition regime with the other regimes. It has a completely different new physical behaviour which in any case cannot be interpreted in pure analytic terms.

Moreover, because of the parity symmetry of Dirac operator, if $\mathrm{A}$ is a nodal gauge field its transformation under parity $A^{P}$ is also a nodal configuration which implies that the singularities are invariant under parity transformation. Thus, the whole non-analytic component $h(A)$ is parity preserving, i.e.

$$
h\left[A^{P}\right]=h[A]
$$

The true source of parity symmetry breaking has a perturbative origin: the induced Chern-Simons term. Although the Chern-Simons radiative corrections can removed by a local counterterm, gauge invariance under large gauge transformation is broken if $k_{R} \notin \mathbb{R}$. Therefore the theory cannot be parity 
invariant and gauge invariant at the same time in this case. Only in the case $k_{R}=2 \pi n$ both symmetries can be simultaneously preserved.

In other terms, because of the hermiticity of $\not D_{A}$ all eigenvalues are real. Thus, the only source of imaginary terms in the effective action comes from negative eigenvalues $-1=\mathrm{e}^{i \pi}$. Now at nodal points one positive eigenvalue becomes negative or one negative eigenvalue becomes positive. Thus, generically, $h(A)$ has a $\pi$ discontinuity at configurations with fermionic zero modes. In the case of fermions in the adjoint representation, there is a level crossing at nodal points between eigenvalues becoming positive and eigenvalues becoming negative (see Figure 3). This explains why in that case the singularities of the effective action do not have any physical effect.
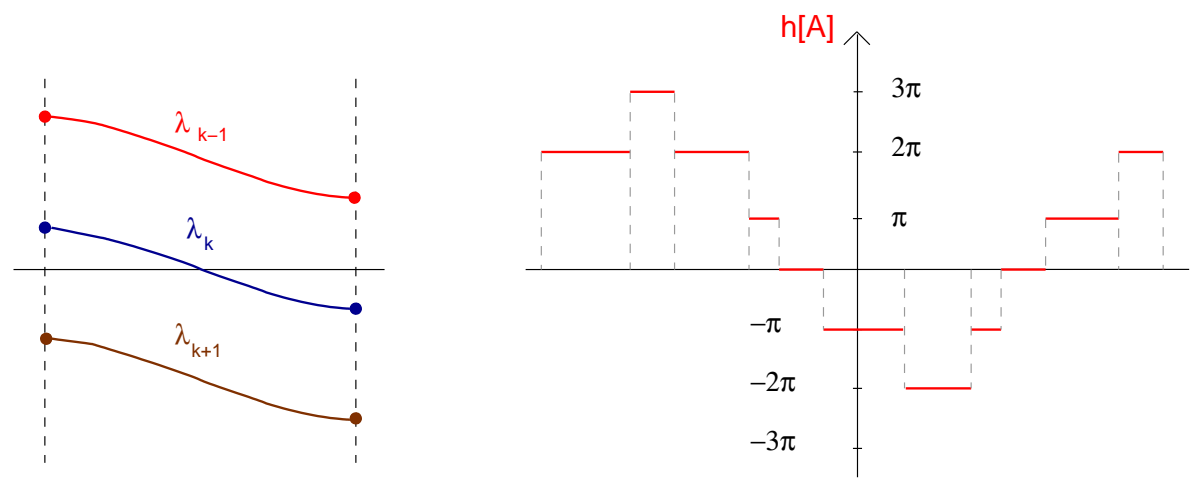

Figure 2. Spectral flow and singular behaviour of the effective action of a fundamental fermionic determinant

In all gauge invariant regularizations the non-analytic term of the effective action $h(A)$ is proportional to $k_{R}-k$ as a consequence of gauge symmetry. This means that the effective counting of zero-level crossings becomes regularization dependent.

In order to illustrate the phenomenon let us consider a lower dimensional example: a fermionic quantum rotor under the action of a magnetic flux [13][32]. In one dimension the equivalent of Chern-Simons action is

$$
k S_{\mathrm{cs}}=k \int A=k \epsilon
$$


and the fermionic determinant of $\not D_{A}=d_{\theta}+A_{\theta}$ can be exactly computed $[32]$

$$
\operatorname{det} \not D_{A}=\mathrm{e}^{-\Gamma(A)}
$$

with

$$
\begin{aligned}
\Gamma(A) & =-\log \left(\left|\cos \frac{\epsilon}{2}\right|+i 2 \pi k_{R}\left[\frac{\epsilon}{2 \pi}+\frac{1}{2}\right]-i \pi k_{R}\right) \\
& =-\log \left[\left|\cos \frac{\epsilon}{2}\right|+i 2 \pi k_{R}\left(\frac{\epsilon}{2 \pi}-\operatorname{Int}\left(\frac{\epsilon}{2 \pi}+\frac{1}{2}\right)\right)\right]
\end{aligned}
$$

with the renormalized coupling constant

$$
k_{\mathrm{R}}= \begin{cases}\frac{1}{2} & \text { if } m>2 n+1 / 2 \\ \frac{1}{\pi} \arctan \frac{\lambda_{+}}{\lambda_{-}} & \text {if } m=2 n+1 / 2 \\ 0 & \text { if } m<2 n+1 / 2\end{cases}
$$

depending on regularization parameters $m, n$ and $\lambda_{+}, \lambda_{-}$. $\operatorname{Int}(x)$ denotes the integer part of $x$. The effective action (5.3), is gauge invariant for any value of these parameters. The Chern-Simons term of the imaginary of the effective action $k_{R} S_{\mathrm{cs}}=k_{R} \epsilon$ is compensated by a non-analytic component

$$
h(A)=-k_{R} \pi\left[2 \operatorname{Int}\left(\frac{\epsilon}{2 \pi}+\frac{1}{2}\right)\right]
$$

which is parity invariant but transforms under global gauge transformations in a way that compensates the anomalous transformation of the ChernSimons part. Notice that the whole imaginary part of $\Gamma(A)$ is proportional to $k_{R}$ in all regularization regimes.

In fermionic determinants the interpretation of singularities in terms of nodal configuration is quite natural. However, in Chern-Simons theory the divergence of $\Gamma_{A}$ at one-loop order is more intriguing because there is not an apriori reason for singularities. In the Schrödinger representation physical states are described by functionals of gauge fields which as pointed out in [33][34] in Chern-Simons theory vanish at certain nodal configurations. It is therefore not unreasonable that the effective action of the theory could diverge at some classical configurations which might be related to nodes of the vacuum state. In general, this type of singularity indicates a suppression 
of tunneling. One can identify some configurations where the one loop effective action diverges. In fact, it is easy to show that there is a discontinuity of $h(A)$ at the sphaleron gauge field on $S^{3}$. This is a gauge field which is a saddle point of Yang-Mills action, i.e. it satisfies the Euclidean Yang-Mills equations and Bianchi identity

$$
D_{A} F(A)=D_{A}^{*} F(A)=0 .
$$

It is given explicitly by

$$
\left[A_{\mathrm{sph}}\right]_{j}=\frac{4 R}{\left(x^{2}+4 R^{2}\right)^{2}}\left(4 R \epsilon_{j k}^{a} x^{k}-2 x^{a} x_{j}+\left[x^{2}-4 R^{2}\right] \delta_{j}^{a}\right) \sigma_{a}
$$

for $S U(2)$ gauge fields ( $R$ is the radius of the $S^{3}$ sphere). The proof that $A_{\mathrm{sph}}$ is a nodal point follows from equations (5.5)which imply that $* F(A)$ is a zero mode of the operator $\Delta_{A}$ which generates the one-loop corrections of the Chern-Simons theory (3.1). There exist a similar phenomenon for massless fermions on the adjoint representation. The sphaleron is a nodal configuration of the corresponding determinant with the same spectral flow. Now, since the fermions are in the adjoint representation, two level cross the zero level at the sphaleron configuration (see Fig. 3).

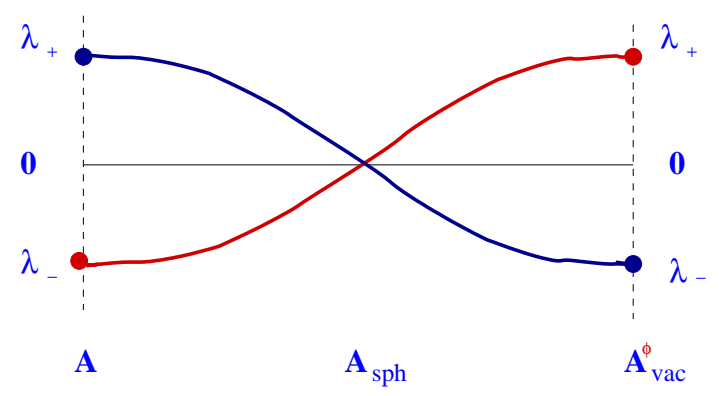

Figure 3. Spectral flow of the fermionic determinant in the adjoint representation

In this case the fermionic determinant $\operatorname{det} \not_{A}$ has the same properties that the vacuum state of $3+1$ dimensional gauge theories at $\theta=\pi$ and the discontinuity of $h(A)$ at sphaleron configurations on $S^{3}$ is a physical property which encodes the tunneling suppression due to the effect of massless fermions [35].

The dependence of the fermionic determinant on the background gauge field contributes to the understanding of the role of singular contributions in 
the effective action. The existence of zero modes determine the existence of discontinuities in the imaginary part of the effective action. In the real part the singularities are more severe. At nodal configurations the real part of the effective action becomes infinite signaling the failure of perturbation theory and the vanishing of the corresponding determinant. The analysis of the physical role of these singularities in Chern-Simons theory and its possible survival at higher orders in the coupling constant $1 / k$ is an open problem.

In order to obtain a better physical picture of the transition regime let us analyse the case of pure Abelian Chern-Simons theory.

$$
S_{\mathrm{cs}}=\frac{k_{R}}{4 \pi} \int A \wedge F(A)
$$

Although in general there is no quantization condition for the coupling constant $k_{R}$, in the presence of magnetic monopoles in $M=S^{1} \times T^{2}$, consistency requires its quantization.

In temporal gauge and flat gauge fields the effective action (5.6) reduces to

$$
S_{\mathrm{cs}}=k_{R} \pi \epsilon^{i j} \int a_{i} \dot{a}_{j}
$$

and can be quantized as a quantum Hall effect in a dual torus $\widehat{T}^{2}$ with magnetic charge $k_{R} \in \mathbb{Z}$. The number of physical states is finite and equals the value of the magnetic charge $k_{R}[36]$. This explains why $k_{R}$ should be quantized.

In the transition regime a massless fermion induces an effective action with $k_{R} \notin \mathbb{Z}$ and extra non-analytic terms in the imaginary part. In order to analyze the physical effect of these terms let us consider a slightly different action with a similar basic behaviour

$$
S_{\mathrm{cS}}=k_{R} \pi \epsilon^{i j} \int\left[a_{i}\right] \dot{a}_{j}
$$

where $[x]=x-\operatorname{Int}(x)$ and $k_{R} \notin \mathbb{Z}$. The system governed by such an action is equivalent to a charged particle moving in a torus under the action of two magnetic fields: one uniform magnetic field with non-integer total magnetic flux $k_{R}$ across the torus, and an extra magnetic field with a deltalike singularity whose magnetic flux just cancels that of the uniform magnetic field.

$$
F_{12}=k_{R} \pi\left[2-\delta\left(a_{1}\right)-\delta\left(a_{2}\right)\right]
$$


Thus, the total magnetic flux is null and gauge invariance under large gauge transformations is restored.

The quantum system has in this case only one vacuum state. Thus, the physical regime associated to transition regularization may be very different from the one obtained from generic regularization schemes. This would explain in physical terms the smooth interpolation between the two generic regularization regimes through the transition regime.

The fact that different regularizations of the theory give rise to different quantum theories is not so surprising. One simple but paradigmatic example is topological quantum mechanics on a Riemann surface $\Sigma$ of genus $h$ in the presence of a magnetic field $A$ with magnetic charge $k$ [33]. In standard Hamiltonian formalism the quantum Hamiltonian is trivial $(H=0)$ as corresponds to a topological theory and the dimension of the space of quantum states is finite and given by $\operatorname{dim} \mathcal{H}_{k}^{0}=1-h+k$, for $k>h-1$. However, if the theory is regularized by means of a metric dependent kinetic term,

$$
L(x, \dot{x})=\frac{1}{2 \Lambda} g_{i j} \dot{x}^{i} \dot{x}^{j}+A_{i} \dot{x}^{i},
$$

the Hamiltonian becomes $H_{\Lambda}=\frac{\Lambda}{2} \Delta_{A}^{g}$, and the topological limit $\Lambda \rightarrow \infty$ is governed by the ground states of $H_{\Lambda}$. The quantum Hilbert space of the topological field theory obtained by this method can have a dimension lower than $1-h+k$, depending on the symmetries of the background metric $g$ of the regularization [33]. In particular, this is the case when the metric $g$ breaks the degeneracy of the ground state of the covariant Laplacian $\Delta_{A}^{g}$. The standard result is obtained by choosing only metrics which is compatible with the magnetic field $B=d A$, in the sense that they give rise to a Kähler structure on $\Sigma$.

\section{A c-theorem in three-dimensions}

The existence of different regimes in the ultraviolet regularization in ChernSimons theory also has relevant implications for the induced gravitational interactions. Although Chern-Simons action is metric independent the quantum corrections generate a finite gravitational Chern-Simons term

$$
S_{\mathrm{csg}}=\frac{\kappa}{4 \pi} \int\left[\epsilon^{\mu \nu \sigma} R_{\mu \nu a b} \omega_{\sigma}^{a b}+\frac{2}{3} \omega_{\mu a}^{b} \omega_{\nu a}^{c} \omega_{\sigma c}^{a}\right] .
$$


This term which gives rise to a metric independent effective action can be canceled by the introduction of a local counterterm. But then a framing anomaly is generated as a physical effect of the theory [10]. The novel effect is that this anomaly also become dependent on the regularization regime as the parity anomaly.

The induced gravitational Chern-Simons term was conjectured to be of the form $\kappa=c / 24$, where $c$ is the central charge of the conformal theory associated to Chern-Simons theory [37]. In the present case, $c=k\left(N^{2}-\right.$ $1) /(k+N)$. In perturbation theory, this means that

$$
\kappa=\frac{N^{2}-1}{24} \sum_{n=0}^{\infty}\left(-\frac{N}{k}\right)^{n} .
$$

However, as anticipated $\kappa$ depends on the choice of regularization regime. The one loop contribution

$$
\kappa_{\mathrm{R}}^{[1]}= \begin{cases}\frac{N^{2}-1}{24} & \text { if } m>2 n+1 / 2 \\ \frac{N^{2}-1}{12 \pi} \arctan \frac{\lambda_{+}}{\lambda_{-}} & \text {if } m=2 n+1 / 2 \\ 0 & \text { if } m<2 n+1 / 2\end{cases}
$$

agrees with the expected value $\kappa=\left(N^{2}-1\right) / 24$ only if $m>2 n+\frac{1}{2}$. The vanishing of $\kappa$ in the regime with $m<2 n+\frac{1}{2}$ was first anticipated by Witten [37]. In this scheme a second order perturbative calculation was carried out in Refs. [38], and the result seems to agree with the standard case. In the transition regime $\kappa$ depends on the weights $\lambda_{+}$and $\lambda_{-}$of the parity odd and parity even regulators and does not correspond to any previously expected behaviourc. In this case there is relation between the value of $\kappa$ and the renormalized Chern-Simons coupling constant $k_{R}[10]$,

$$
\kappa=\frac{\left(k_{R}-k\right)\left(N^{2}-1\right)}{24 N} .
$$

The above results suggest that this relation holds for the three regimes. It would be very interesting to investigate if the property also holds beyond one loop approximation.

Other types of gravitational terms like Einstein or cosmological constant terms can also be generated in the effective action, but they present linear

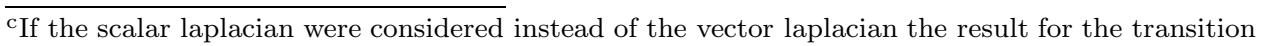
regime will be different 
or cubic UV divergences which need to be renormalized leaving an extra ambiguity in the actual values of the corresponding renormalized couplings. Metric independence requires the cancellation of both couplings. But the same gravitational Chern-Simons term also contains some hidden Einstein and cosmological terms when the gauge field is written in terms of the vierbein and the spin connexion [39]. The different values of the renormalized gravitational Chern-Simons constant also adds an extra source of metric dependence. Although the induced Chern-Simons term can be removed by a choice of renormalization scheme its non-analytic counterpart cannot and in fact yields an extra frame dependent contribution. Only the third regime provides a fully consistent metric independent theory without parity and framing anomalies.

This connection between the renormalization of Chern-Simons coupling and the induced gravitational Chern-Simons coefficient ant its relation to the central charge of the associated conformal theory suggests a possible extension of Zamolodchikov's c-theorem to three-dimensional systems. Topological Chern-Simons theories would correspond to two dimensional conformal theories and the interpolating regularized topologically massive theories will generate a flow from one theory with one Chern-Simons coupling to another with a different one. A c-theorem would establish the existence of a monotone function along this renormalization group flow which will coincide with the coupling of gravitational Chern-Simons term at topological fixed points. One natural candidate for Zamolodchivov c-function, thus, can be defined in terms of the induced gravitational Chern-Simons term which is identical to $\kappa$ at the pure Chern-Simons theories and varies along renormalization group trajectories. A concrete proposal based on an version of Zamolodchikov theorem formulated in Ref. [40] can be established from the following spectral 
representation of the stress tensor correlators

$$
\begin{aligned}
& \left\langle T_{\alpha \beta}(x) T_{\mu \nu}(0)\right\rangle_{\text {odd }}= \\
& -\frac{1}{192 \pi} \int d^{3} x \frac{\mathrm{e}^{i p . x}}{(2 \pi)^{3 / 2}} \int_{0}^{\infty} d \lambda \frac{\lambda c(\lambda)}{\sqrt{p^{2}+\lambda^{2}}} \\
& {\left[\epsilon_{\mu \sigma \alpha} p^{\sigma}\left(p_{\nu} p_{\beta}-\delta_{\nu \beta}\right)+\epsilon_{\nu \sigma \alpha} p^{\sigma}\left(p_{\mu} p_{\beta}-\delta_{\mu \beta}\right)+\right.} \\
& \left.\epsilon_{\nu \sigma \beta} p^{\sigma}\left(p_{\mu} p_{\alpha}-\delta_{\mu \alpha}\right)+\epsilon_{\mu \sigma \beta} p^{\sigma}\left(p_{\nu} p_{\alpha}-\delta_{\nu \alpha}\right)\right]
\end{aligned}
$$

$c(\lambda)$ emerges as a natural candidate for a Zamolodchikov c-function for threedimensional theories. Unfortunately, $c(\lambda)$ cannot be universally monotone for the same reasons as the similar spectral representation of the flow of the effective $k$ coupling cannot be monotone in all regularization regimes of pure Chern-Simons theory (see Fig.1) d . This negative result does not exclude the existence of another extension of c-theorem to $2+1$ dimensional theories. It merely points out that the spectral representation of the gravitational Chern-Simons term is not a good c-function. On the other hand for purely bosonic theories there are not axial Chern-Simons like interactions which could generate a simple way of describing the irreversibility of the renormalization group flow.

\section{Discussion}

The presence of non-analytic terms in the effective action is fundamental for the right physical description of Chern-Simons theory and massless fermions in $2+1$ dimensions. The existence of such contributions is pointed out by the appearance of different perturbative corrections in different gauge invariant regularizations. The discontinuities associated to those non-analytic terms signal the presence of physical singularities associated to the zeros of the partition function in some backgrounds. The appearance of nodal configurations is also related to the quantum tunneling suppression. In massless fermions nodal contributions are associated to the existence of fermionic zero

d This observation was made by Ian Kogan and one of us (M.A.) 
modes. However, the structure of singularities and discontinuities depends on the regularization regime and makes possible the physical differences between the corresponding theories. In regularizations with transition regimes the nature of the singularities associated to non-analytic terms suggest a non holomorphic behaviour of the effective partition function in terms of classical fields. It is remarkable that it is possible to find out gauge invariant regularization regimes where there are not parity and framing anomalies. This suggests that those anomalies can be better understood as spontaneous symmetry breaking phenomena rather than genuine anomalies. In some toy models it has been shown that the transition regularizations keep constant the number of physical states. It would be very interesting to analyze the behaviour of the number of physical states of the Chern-Simons theory in the transition regime and verify if it is dramatically reduced as in the toy model. Finally, it is pointed out why the extension of Zamolodchikov c-theorem to three-dimensional theories is an interesting still open problem.

\section{Acknowledgments}

We thank Fernando Falceto, Gloria Luzón and very specially Ian Kogan for enlightening discussions during different stages of the realization of the paper. This work is partially supported by CICYT (grant FPA2004-02948).

\section{References}

1. J. Schonfeld, Nucl. Phys. B 185 (1981) 157 S. Deser, R. Jackiw, S. Templeton, Phys. Rev. Lett. 48 (1982) 975; Ann. Phys. (N.Y.) 140 (1982) 372

2. R. Jackiw, In Gauge Theories of the Eighties, Eds. E. Ratio, J. Lindfords, Lecture Notes in Physics, vol. 181, Springer (1983)

3. M. Asorey, P.K. Mitter, Phys. Lett. B153 (1985) 147

4. R. D. Pisarski, S. Rao, Phys. Rev. D 32 (1985) 2081

5. W. Chen, G. W. Semenoff, Y.-S. Wu, Mod. Phys. Lett. A5 (1990) 1833

6. L. Alvarez-Gaumé, J.M.F. Labastida and A.V. Ramallo, Nucl. Phys. B334 (1990) 103

7. M. Asorey, F. Falceto, Phys. Lett. B 241 (1990) 31

8. C.P. Martin, Phys. Lett. B 241 (1990) 513

9. M. A. Shifman, Nucl. Phys. B352 (1991) 87

10. M. Asorey, F. Falceto, J.L. López and G. Luzón, Nucl. Phys. B429 (1994) 344.

11. M. Atiyah, V. Patodi, I.M. Singer, I. Math. Proc. Comb. Phil. Soc. 77 (1975) 43 and II 78 (1975) 405

12. A.V. Redlich, Phys. Rev. Lett. 52 (1981) 18; Phys. Rev. D29 (1984) 2366.

13. G. Dunne, K. Lee and Ch. Lu, Phys. Rev. Lett. 78 (1997) 3434 
14. C. Fosco, G.L. Rossini and F. A. Schaposnik, Phys. Rev. Lett. 79 (1997) 1980

15. S. Deser, L. Griguolo and D. Seminara, Phys. Rev. Lett. 79 (1997) 1976; Phys. Rev. D 57 (1998) 7444

16. R. Jackiw and Y.S. Pi, Phys. Lett. B 423 (1998) 364.

17. E. Witten, Commun. Math. Phys. 121 (1989) 351.

18. D. Bar-Natan, E. Witten, Commun. Math. Phys. 141 (1991) 423

19. K. Gawedzki and A. Kupiainen, Phys. Lett. B215 (1988) 119; Nucl. Phys. B320 (1989) 649

20. A. Slavnov, Theor. Math. Phys. 33 (1977) 210

21. M.Asorey and F. Falceto, unpublished (1990)

22. G. P. Korchemsky, Mod. Phys. Lett. A6 (1991) 727

23. T. Kimura, Prog. Theor. Phys. 92 (1994) 693.

24. R. Narayanan and J. Nishimura, Nucl. Phys. B 508 (1997) 371.

25. A. Slavnov, Phys. Lett. B415 (1997) 390

26. E. Witten,Phys. Lett. B 117 (1982) 324.

27. D. Karabali and V.P. Nair, Nucl. Phys. B464 (1996) 135; Phys. Lett. B379 (1996) 141; Int. J. Mod. Phys A12 (1997) 1161.

28. M. Teper, Phys. Lett. B311 (1993) 223; O. Philipsen, M. Teper and H. Wittig, Nucl. Phys. B469 (1996) 445 ; M. Teper, Phys. Rev. D59 (1999) 014512

29. M. F. Atiyah and I.M. Singer, Ann. of Math. 87 (1968) 485, 546; 93 (1971) 1, 119, 139; M. F. Atiyah and G.B. Segal, Ann. of Math. 87 (1968) 531.

30. L. Alvarez-Gaumé, S. Della Pietra and G. Moore, Ann. Phys. 163 (1985) 288.

31. D. Seminara, In Particles, Strings and Cosmology, Ed. P. Nath, World Sci., Singapore (1999)

32. J.L. López, J. Phys. A 31 (1998) 7955

33. M. Asorey, J. Geom. Phys. 11(1993) 63

34. M. Asorey, F. Falceto, and G. Luzón, Phys. Lett. B 349 (1995) 125

35. M. Asorey and F. Falceto, Phys. Rev. Lett. 77(1996) 3074

36. M. Bos, V.P. Nair, Phys. Lett. B 223 (1989) 61

37. E. Witten, in Physics and Mathematics of Strings, L. Brink ed., World Sci., Singapore (1990).

38. S. Axelrod, I.M. Singer, In Proceedings Differential geometric methods in theoretical physics, vol. 1, S. Catto and A. Rocha, eds., World Sci, Singapore (1992) 3; Diff. Geom. 39 (1994) 173

39. A. Achúcarro and P. Townsend,Phys. Lett. B 180 (1986)383

40. A. Capelli, D. Friedan and J. I. Latorre, Nucl. Phys. 352 (1991) 616 